\title{
Chandra observations of nuclear X-ray emission from a sample of radio sources
}

\author{
J. K. Gambill ${ }^{1}$, R. M. Sambruna ${ }^{1,2}$, G. Chartas ${ }^{3}$, C. C. Cheung ${ }^{4}$, L. Maraschi ${ }^{5}$, F. Tavecchio ${ }^{5}$, \\ C. M. Urry ${ }^{6}$, and J. E. Pesce ${ }^{2}$
}

1 School of Computational Sciences, MS 5C3, George Mason University, 4400 University Drive, Fairfax, VA 22030, USA

2 Department of Physics and Astronomy, MS 3F3, George Mason University, 4400 University Drive, Fairfax, VA 22030, USA

3 Department of Astronomy and Astrophysics, 525 Davey Lab, The Pennsylvania State University, State College, PA 16802, USA

4 Department of Physics, MS 057, Brandeis University, Waltham, MA 02454, USA

5 Osservatorio Astronomico di Brera, via Brera 28, Milan 20121, Italy

${ }^{6}$ Department of Astronomy, PO Box 208101, Yale University, New Haven, CT 06520, USA

Received 14 November 2002 / Accepted 30 January 2003

\begin{abstract}
We present the X-ray properties of a sample of 17 radio sources observed with the Chandra X-ray Observatory as part of a project aimed at studying the X-ray emission from their radio jets. In this paper, we concentrate on the X-ray properties of the unresolved cores. The sample includes 16 quasars (11 core-dominated and 5 lobe-dominated) in the redshift range $z=0.30-1.96$, and one low-power radio-galaxy at $z=0.064$. No diffuse X-ray emission is present around the cores of the quasars, except for the nearby low-power galaxy that has diffuse emission on a scale and with a luminosity consistent with other FRIs. No high-amplitude, short-term variability is detected within the relatively short Chandra exposures. However, 1510-089 shows low-amplitude flux changes with a timescale of $\sim 25 \mathrm{~min}$. The X-ray spectra of the quasar cores are generally well described by a single power law model with Galactic absorption. However, in six quasars we find soft X-ray excess emission below $1.6 \mathrm{keV}$. Interestingly, we detect an $\mathrm{Fe} \mathrm{K}$-shell emission line, consistent with fluorescent $\mathrm{K} \alpha$ emission from cold iron, in one lobe- and two core-dominated sources. The average X-ray photon index for the quasars in the sample is $\Gamma_{\text {sample }}=1.66$ and dispersion $\sigma_{\text {sample }}=0.23$. The average spectral slope for our sample is flatter than the slope found for radio-quiet quasars and for radio-loud AGNs with larger jet orientations; this indicates that beaming affects the X-ray emission from the cores in our sample of quasars.
\end{abstract}

Key words. galaxies: active - radio continuum: galaxies - galaxies: quasars: emission lines - X-rays: general

\section{Introduction}

An important distinction in the quasar class is between radioquiet and radio-loud active galactic nuclei (AGNs), based on their optical-to-radio flux ratios (e.g., Kellermann et al. 1989). While the ultimate source of power is thought to be the same for both classes of AGNs (i.e., accretion of gas onto a supermassive black hole; Antonucci 1993; Urry \& Padovani 1995), there are subtle but systematic differences in the continuum and line properties from radio to optical/UV wavelengths between the classes (Sanders et al. 1989; Miley \& Miller 1979; Yee \& Oke 1978). X-ray observations of both types of sources probe the inner regions and can thus help discriminate the origin of the radio-loud/quiet difference. However, while radio-quiet quasars have been extensively studied at X-rays (e.g., George et al. 2000; Fiore et al. 1998; Nandra et al. 1997a,b), the X-ray properties of radio-loud AGNs are relatively less well known

Send offprint requests to: J. K. Gambill,

e-mail: jessica@physics.gmu.edu (e.g., Sambruna et al. 2002a and references therein). The study of the nuclear X-ray emission from radio-loud quasars with previous X-ray satellites was generally hampered by poor angular resolution and/or sensitivity.

Earlier studies of AGNs at medium-soft X-rays with Einstein and ROSAT showed that radio-loud quasars have significantly flatter continuum slopes, $\Gamma \sim 1.5$, than their radioquiet counterparts, $\Gamma \sim 2.0$ (Fiore et al. 1998; Nandra et al. 1997a,b; Shastri 1991; Wilkes \& Elvis 1987). This was attributed to dilution of the core emission by a beamed X-ray component from the jet. Recent systematic studies of radioloud AGNs at harder X-rays with ASCA confirmed that radioloud quasars are flatter, $\Gamma \sim 1.6$, than radio-quiet quasars (Reeves \& Turner 2000). However, observations of Broad-Line Radio Galaxies (BLRGs) and double-lobe quasars, believed to be at larger jet orientations, indicate steeper slopes $(\Gamma \sim 1.8)$ more similar to Seyferts (Hasenkopf et al. 2002; Sambruna et al. 1999). 
In low-luminosity radio-quiet AGNs, Fe K-shell emission is a relatively well known property. In several Seyfert 1s a prominent $\mathrm{K} \alpha$ fluorescent emission line from cold $\mathrm{Fe}$ was detected with $A S C A$ at rest-frame energies $6.4 \mathrm{keV}$, with Equivalent Widths $(E W \mathrm{~s}) \sim 250 \mathrm{eV}$ (Nandra et al. 1997b). However, the presence and the nature of $\mathrm{Fe} \mathrm{K}$ emission at higher luminosities is not yet established. Nandra et al. (1997a) detected an $\mathrm{Fe}$ line in a radio-quiet quasar at energies higher than $6.4 \mathrm{keV}$, consistent with emission from highly ionized iron. A highly ionized Fe line, observed by ASCA with energies consistent with emission from Fe XXV-XXVI, may be common in radio-quiet quasars (Reeves \& Turner 2000). An ionized Fe line was recently confirmed in a high-redshift quasar with XMM-Newton (Reeves et al. 2001).

However, Fe K emission is rare in radio-loud AGNs. So far, a weak and generally narrow $\mathrm{Fe} \mathrm{K} \alpha$ emission line was observed in the brightest BLRGs (see Sambruna et al. 2002a and references therein), and more recently in 3 high-luminosity $\left(L_{2-10 \mathrm{keV}} \sim 10^{45} \mathrm{ergs} \mathrm{s}^{-1}\right)$ radio-loud quasars (Hasenkopf et al. 2002). While there is evidence that $\mathrm{Fe} \mathrm{K}$ emission and other reflection features in BLRGs are intrinsically weak (e.g., Eracleous et al. 2000), an additional difficulty in their detection is the presence of diffuse X-ray emission from the host galaxy/associated cluster of galaxies, which may dilute any intrinsic (weak) Fe emission lines from the active nucleus in lowresolution observations.

The advent of the Chandra X-ray Observatory, with unprecedented angular resolution $\left(0.492^{\prime \prime} /\right.$ pixel) and improved sensitivity, makes significant progress possible in the study of the X-ray emission from the cores of radio-loud quasars. With Chandra, the X-ray emission from the nucleus can be isolated from the extended (kiloparsec-scale) jet emission and other extended components (e.g., thermal radiation from a cluster). Also, the X-ray spectral index and other features in the spectrum can be better quantified.

In this paper, we report on the X-ray properties of the nuclei of 17 radio-loud sources observed with Chandra. The sample was selected based on the properties of the extended radio features as part of a survey aimed at finding the jet $\mathrm{X}$ ray and optical counterparts with Chandra and the Hubble Space Telescope (HST). The Chandra and multiwavelength jet properties of the first six observed sources were discussed in Sambruna et al. (2002b), while the remaning sources will be presented in a future publication. Here we concentrate on the $\mathrm{X}$-ray properties of the cores in our Chandra images.

The plan of the paper is as follows: Sect. 2 presents the sample, the Chandra observations, and the analysis methods, Sect. 3 describes the results of the spatial, timing, and spectral analysis, and Sect. 4 discusses the implications of Sect. 3. Throughout this work, $H_{0}=75 \mathrm{~km} \mathrm{~s}^{-1} \mathrm{Mpc}^{-1}$ and $q_{0}=0.5$ are adopted.

\section{Observations}

\subsection{The sample}

The selection criteria of the sample of 17 radio-loud sources are discussed in Sambruna et al. (2002b). Briefly, the sample was extracted from the list of radio jets of Bridle \& Perley (1984) and Liu \& Xie (1992) based on the length and brightness of the jet, in order to match the Chandra and HST observing capabilities. As a result, the sample contains 17 targets and is biased toward the presence of a bright radio jet. The targets include 16 quasars, 11 Flat Spectrum Radio Quasars (FSRQs), 5 Steep Spectrum Radio Quasars (SSRQs), and 1 nearby, lowpower radio galaxy $(0836+299)$. All the quasars have one-sided jets indicating that beaming is likely to be important in these sources.

Chandra and HST observations were awarded to us for 16 of the 17 sources in the sample. Chandra observations of the 17th source, 3C 207, were awarded to other investigators (Brunetti et al. 2002); we complete our sample with archival Chandra data of 3C 207. Table 1 lists the sources and their basic properties. Based on the listed ratio of core-to-jet radio power (Cheung et al. 2003), 11 sources are classified as coredominated and 6 as lobe-dominated (Table 1, Col. 7).

All sources except $0836+299$ are high-power radio sources $\left(P_{1.4 \mathrm{GHz}} \gtrsim 10^{32} \mathrm{ergs} \mathrm{s}^{-1}\right)$ and exhibit a Fanaroff-Riley II (FRII) radio morphology (Fanaroff \& Riley 1974). The source $0836+299$ has low-power $\left(P_{1.4 \mathrm{GHz}} \sim 3 \times 10^{31} \mathrm{ergs} \mathrm{s}^{-1}\right)$ and a radio morphology resembling a Fanaroff-Riley I (FRI) (van Breugel et al. 1986). We classify this source as an FRI.

\subsection{Data acquisition, reduction, and analysis}

The observations were made with the Advanced CCD Imaging Spectrometer (ACIS-S), with the sources at the nominal aimpoint on chip S3. The awarded exposures were $10 \mathrm{ks}$ per target, with occasionally longer or shorter exposures to accommodate gaps in the Chandra observing schedule. To avoid pileup of the core, a 1/8 subarray mode was used with one operational CCD, reducing the frame time to $0.4 \mathrm{~s}$. Despite this precaution, the nuclear X-ray emission was so strong that in 10/17 sources pileup was still present. The measured counts/frame for the piledup sources range from 0.1 to 0.5 counts/frame. Based on Fig. 6.25 of the Chandra Proposer Observer Guide (POG), the pileup fraction in the sources of our sample ranges between $1 \%$ and $10 \%$. Pileup is expected to produce significant changes to the spectral slopes $(\delta \Gamma<-0.1)$ for observed count rates greater than 0.1 counts/frame.

The Chandra data were reduced using CIAO v. 2.1.2 and following standard criteria, using the latest calibration files provided by the Chandra X-ray Center (CXC). Pixel randomization was removed and only events for ASCA grades 0, 2-4, and 6 were retained for the analysis. The data were further restricted to the energy range $0.5-8 \mathrm{keV}$, where the background is negligible and the ACIS-S calibration is best known. We also checked that no flaring background events occurred during the observations. After screening, the effective exposure times range between 8.4-10.4 ks, with one longer exposure, $37.5 \mathrm{ks}$ $(0838+133)$. Table 2 lists the details of the Chandra observations, where a flag has been added to denote the sources affected by pileup (Col. 5).

For the seven sources not affected by pileup (unpiled sources), X-ray fluxes and spectra were extracted using a circular region with a $2^{\prime \prime}$ radius centered on the brightest pixel. 
Table 1. Sample sources and their basic properties.

\begin{tabular}{clccccc}
\hline \hline $\begin{array}{c}\text { Source } \\
(1)\end{array}$ & $\begin{array}{c}\text { Alt name } \\
(2)\end{array}$ & $\begin{array}{c}m_{V} \\
(3)\end{array}$ & $\begin{array}{c}z \\
(4)\end{array}$ & $\begin{array}{c}N_{\mathrm{H}}^{\text {Gal }} \\
(5)\end{array}$ & $\begin{array}{c}\text { Type } \\
(6)\end{array}$ & $\begin{array}{c}R_{i} \\
(7)\end{array}$ \\
\hline $0405-123$ & PKS & 14.8 & 0.574 & 3.81 & FSRQ & 1.1 \\
$0605-085$ & PKS & 18.5 & 0.870 & 21.0 & FSRQ & 14.5 \\
$0723+679$ & 3C 179 & 18.0 & 0.846 & 4.31 & SSRQ & 0.44 \\
& 4C +67.14 & & & & & \\
$0802+103$ & 3C 191 & 18.4 & 1.956 & 2.28 & SSRQ & 0.03 \\
& 4C +10.25 & & & & & \\
$0836+299$ & 4C +29.30 & 15.7 & 0.064 & 4.06 & RG & 0.06 \\
$0838+133$ & 3C 207 & 18.5 & 0.684 & 4.00 & SSRQ & 0.43 \\
& 4C +13.38 & & & & & \\
$1040+123$ & 3C 245 & 17.3 & 1.029 & 2.87 & SSRQ & $1.1^{a}$ \\
& 4C +12.37 & & & & & \\
$1055+018$ & 4C +01.28 & 18.3 & 0.888 & 3.40 & FSRQ/BL & 19.2 \\
$1136-135$ & PKS & 16.1 & 0.554 & 3.59 & SSRQ & 0.29 \\
$1150+497$ & 4C +49.22 & 17.1 & 0.334 & 2.05 & FSRQ & 2.4 \\
$1354+195$ & 4C +19.44 & 16.0 & 0.720 & 2.18 & FSRQ & 2.7 \\
$1510-089$ & PKS & 16.5 & 0.361 & 7.96 & FSRQ & 6.7 \\
$1641+399$ & 3C 345 & 16.0 & 0.594 & 1.13 & FSRQ & 9.1 \\
& 4C +39.48 & & & & & \\
$1642+690$ & 4C +69.21 & 19.2 & 0.751 & 4.54 & FSRQ & 6.8 \\
$1741+279$ & 4C +27.38 & 17.7 & 0.372 & 4.59 & FSRQ & 1.6 \\
$1928+738$ & 4C +73.18 & 16.5 & 0.302 & 7.71 & FSRQ & 16.8 \\
$2251+134$ & 4C +13.85 & 19.5 & 0.673 & 4.98 & FSRQ & 1.1 \\
\hline
\end{tabular}

Columns explanation: 1 = Source IAU name; 2 = Common source names; 3 = Optical magnitude, $V$-band; $4=$ Redshift; $5=$ Galactic column density in units $10^{20} \mathrm{~cm}^{-2}$ from NED Galactic Extinction Calculator; 6 = Source type. FSRQ: Flat Spectrum Radio Quasar; SSRQ: Steep Spectrum Radio Quasar; RG: Radio Galaxy; BL: BL Lac Object; 7 = Ratio of core to extended radio power at $5 \mathrm{GHz}$, corrected for the redshift (observed value $\times(1+z)^{-1}$ ) from Cheung et al. (2003). Core-dominated sources have $R_{i}>1$, while lobe-dominated sources have $R_{i}<1$.

Notes: $a=$ The quasar $1040+123$ is known to be a lobe-dominated source with a variable nucleus (Hough \& Readhead 1989). However, this core was brighter than it had been previously during the epoch of our archival observation, hence the $R_{i}$ value greater than unity. We classify this object as a lobe-dominated SSRQ in our discussion, as previous observations have established (Hough \& Readhead 1989).

Based on the ACIS-S encircled energy fraction (Fig. 6.3 in the Chandra POG), a source extraction radius of $1^{\prime \prime}$ encircles $\gtrsim 90 \%$ of the flux of a point source at $1 \mathrm{keV}$. From this guideline and with our findings in the spatial analysis (Sect. 3.1, i.e., the radial profiles drop to zero around $2^{\prime \prime}$ ), we consider a source extraction region of $2^{\prime \prime}$ to contain all of the core flux. Smaller extraction regions (radius $=1.5^{\prime \prime}$ ) were used for two objects $(0802+103$ and $0836+299)$ since the $2^{\prime \prime}$ aperture included emission from the X-ray jets. The background was estimated in a nearby region free of obvious sources, with a radius of $8^{\prime \prime}$. The net source counts in $0.5-8 \mathrm{keV}$ after background subtraction are reported in Table 2. We estimated the total Xray counts in the jet, by extracting the counts from a rectangular region of varying widths and heights to best suit the shape of the particular jet; the dimensions of the box are reported in Table 2. Also listed in Table 2 is the ratio of the core-tojet X-ray counts (Col. 7); this ratio can be used to gauge the contribution of the jet to the total X-ray emission in previous, lower-resolution X-ray observations.

For the 10 sources affected by pileup of the nuclear flux, we list in Table 2 the total counts collected in a $2^{\prime \prime}$ radius circle. Since pileup is known to affect the spectral index, we extracted
X-ray spectra for these objects using the counts in the wings of the PSF. For relatively low pileup rates, pileup is not believed to affect the wings of the PSF, only its core (within $0.5^{\prime \prime}$ ). We extracted the spectrum using an annulus centered on the source centroid with inner radius $R_{\text {in }}$ and outer radius fixed at $R_{\text {out }}=3^{\prime \prime}$. We experimented with various choices of $R_{\text {in }}$ (from $0.5^{\prime \prime}$ to $1.5^{\prime \prime}$ ), and selected the radius that gave the maximum number of counts for spectral analysis, $R_{\text {in }}=0.5^{\prime \prime}$. The ancillary files were corrected for the chosen annular extraction region, and used for the spectral fits.

\subsubsection{Spatial analysis}

Spatial analysis was performed on all 17 sources. Our aim was to investigate whether diffuse $\mathrm{X}$-ray emission is present around the cores, or whether the X-ray radial profile is entirely consistent with the instrumental point spread function (PSF).

The instrumental PSFs were created using the PSF library for the ACIS camera at $1.5 \mathrm{keV}$, from the Chandra Calibration Database (CALDB). The CIAO tool mkpsf simulates a point source, and the tool dmimgcalc normalizes the instrumental PSF to the count rate of a specific nucleus. The extraction of 
Table 2. Log of Chandra observations.

\begin{tabular}{ccccccc}
\hline \hline $\begin{array}{c}\text { Source } \\
(1)\end{array}$ & $\begin{array}{c}\text { Start date } \\
(2)\end{array}$ & $\begin{array}{c}\text { Exposure } \\
(3)\end{array}$ & $\begin{array}{c}\text { Net counts } \\
(4)\end{array}$ & $\begin{array}{c}\text { Pileup } \\
(5)\end{array}$ & $\begin{array}{c}\text { Box } \\
(6)\end{array}$ & $\begin{array}{c}\text { Ratio } \\
(7)\end{array}$ \\
\hline $0405-123$ & $2001-07-22$ & 8661.6 & 10445 & $\mathrm{Y}$ & $2.5 \times 20$ & 120 \\
$0605-085$ & $2001-05-01$ & 8663.2 & 1254 & $\mathrm{~N}$ & $4.0 \times 3.0$ & 16 \\
$0723+679$ & $2001-01-15$ & 9333.3 & 1820 & $\mathrm{~N}$ & $6.0 \times 3.0$ & 54 \\
$0802+103$ & $2001-03-07$ & 8318.9 & $253^{a}$ & $\mathrm{~N}$ & $3.0 \times 1.0$ & 0.9 \\
$0836+299$ & $2001-04-08$ & 7690.9 & $80^{a}$ & $\mathrm{~N}$ & $4.0 \times 18$ & 0.3 \\
$0838+133$ & $2000-11-04$ & 37,544 & 5872 & $\mathrm{Y}$ & $6.0 \times 3.0$ & 20 \\
$1040+123$ & $2001-02-12$ & 10402. & 1956 & $\mathrm{~N}$ & $3.0 \times 4.0$ & 92 \\
$1055+018$ & $2001-01-09$ & 9314.3 & 5887 & $\mathrm{Y}$ & $2.0 \times 4.0$ & 370 \\
$1136-135$ & $2000-11-30$ & 8906.2 & 3162 & $\mathrm{Y}$ & $5.0 \times 9.0$ & 22 \\
$1150+497$ & $2000-12-10$ & 9293.7 & 7612 & $\mathrm{Y}$ & $4.0 \times 8.0$ & 38 \\
$1354+195$ & $2001-01-08$ & 9055.7 & 5624 & $\mathrm{Y}$ & $3.0 \times 30$ & 60 \\
$1510-089$ & $2001-03-23$ & 9241.4 & 5988 & $\mathrm{Y}$ & $6.0 \times 8.0$ & 31 \\
$1641+399$ & $2001-04-27$ & 9055.7 & 6076 & $\mathrm{Y}$ & $5.0 \times 4.0$ & 82 \\
$1642+690$ & $2001-03-08$ & 8326.9 & 1382 & $\mathrm{~N}$ & $4.0 \times 4.5$ & 44 \\
$1741+279$ & $2001-07-21$ & 8895.3 & 2224 & $\mathrm{Y}$ & $5.0 \times 4.0$ & 115 \\
$1928+738$ & $2001-04-27$ & 8392.5 & 6899 & $\mathrm{Y}$ & $6.0 \times 4.0$ & 87 \\
$2251+134$ & $2000-10-21$ & 9185.6 & 1699 & $\mathrm{~N}$ & $7.0 \times 7.0$ & 183 \\
\hline
\end{tabular}

Columns explanation: 1 = Source IAU name; 2 = Observation start date (Year-Month-Day); 3 = Net Chandra exposure, in seconds; 4 = Core photon counts in the energy range $0.5-8 \mathrm{keV}$ from a $2^{\prime \prime}$ apeture; $5=$ Flag indicating pileup of the Chandra data; $6=$ Dimensions of box used to extract the jet count rate, width $\times$ length, ("); $7=$ Ratio of core-to-jet counts.

Notes: $a=$ Core region is defined by a $1.5^{\prime \prime}$ extraction radius (see text Sect. 2.2).

radial profiles allows the data from this normalized image to be compared to the observations.

Radial profiles were extracted using the CIAO tools dmextract and dmtcalc, after restricting the images to the energy range of Chandra sensitivity, $0.5-8 \mathrm{keV}$, and cleaning them of features that would contaminate the radial profiles. Specifically, serendipitous X-ray sources in the field (as for 0605-085, 1136-135) were removed, as well as Xray counterparts of extended radio features (Table 2, Col. 6). Additionally, for piledup sources (Table 2, Col. 5), the charge transfer trail from the nucleus was removed. The CIAO tools dmextract and dmtcalc only calculate a monochromatic PSF, while it is well known that the ACIS PSF is energydependent. Following Donato et al. (2003) and Worrall et al. (2001), we calculated PSF profiles at eight different energies $(0.73,0.98,1.23,1.63,2.13,3.03,5.48,7.23 \mathrm{keV})$ and assigned a weight according to the distribution of photons as a function of energy. The PSF used for comparison with the observed radial profiles is the weighted sum of the energy-dependent PSFs.

Upon the cleaned images, centered on the source centroid, forty annuli of equal width $\left(0.5^{\prime \prime}\right)$ were placed in order to extract the radial profiles out to $20^{\prime \prime}$. The background was extracted from an annular region, centered on the source centroid with an inner radius $R_{\text {in }}=21^{\prime \prime}$ and an outer radius $R_{\text {out }}=26^{\prime \prime}$, outside the region of the profile. For the nearby FRI radio galaxy $0836+299$, the radial profiles were extracted from wider annuli (1.25" radius) up to $25^{\prime \prime}$ from the core. The background for this object was extracted from an annular region with an inner radius $R_{\text {in }}=26^{\prime \prime}$ and an outer radius $R_{\text {out }}=31^{\prime \prime}$. This is the maximum distance allowed in the $1 / 8$ subarray mode. For sources affected by core pileup we normalized the PSF to the observed radial profile at the fourth pixel, (as suggested by the Chandra POG) otherwise, the final PSF was normalized to the first pixel.

\subsubsection{Timing analysis}

To check for time variability of the source flux within the Chandra exposure, we extracted light curves of the cores using the FTOOL "lcurve" v. 1.0 (XRONOS 5.16). Light curves were extracted from the $2^{\prime \prime}$ radius core region in the $0.5-8 \mathrm{keV}$ energy range. The light curves were plotted at a variety of binnings (100-750 s intervals) to check for patterns that might suggest short-timescale variability. To decide if variability was present, we performed a standard $\chi^{2}$ test. We tested for significant deviations from the average count rate within the light curves. The value for $\chi^{2}$ varied with the different binnings but not significantly. Listed in Table 3 are the $\chi^{2}$ values calculated from the $500 \mathrm{~s}$ bins and the probability $P_{\chi^{2}}$ at this binning. This is the probability that the light curve is constant.

\subsubsection{Spectral analysis}

Spectral analysis was performed within XSPEC v.11.0.1. To account for the recently observed quantum efficiency decay of ACIS, possibly caused by molecular contamination of the ACIS filters, we have applied a time-dependent correction to the ACIS quantum efficiency based on the presently available information from the CXC. The fits were restricted to the energy range $0.5-8.0 \mathrm{keV}$, where the background contribution is negligible and where the ACIS-S calibration is best known, especially regarding the ACIS-S gain.

Generally, we performed spectral analysis only for sources where 100 counts or more were detected from the core (unpiled 
sources) or from the PSF wings (piledup sources; see above). The spectra were rebinned to have a minimum of 20 counts in each new bin, in order to apply the $\chi^{2}$ statistics. For the lowpower source $0836+299$, only 80 counts were detected from the core. Although this is formally less than our threshold number of counts for spectral analysis, inspection of the distribution of counts versus energy in this source reveals an interesting spectrum (see below). In light of our interest in this source (the only low-power, FRI source of our sample), we performed spectral analysis for $0836+299$ with a different binning ( $15 \mathrm{cts} / \mathrm{bin})$.

To begin, we fitted all sources with a single power law model with an absorption column density fixed to the Galactic value (Table 1, Col. 5), using solar abundances and the Morrison \& McCammon (1983) cross sections. Next, several multi-component models were fitted to the ACIS spectra of the nuclei, until the minimum $\chi^{2}$ was obtained. The significance of adding more complex models to the fit was estimated via the F-test probability over the original fit. We report both the simple power law fits and the significant multi-component fits.

\section{Results}

Below we present the results from the spatial, timing, and spectral analysis for the whole sample. Tables 3-7 report the results in numerical form.

\subsection{Spatial properties}

We find that in $16 / 17$ sources there is no evidence for diffuse $\mathrm{X}$-ray emission around the cores in the short Chandra exposures: the background-subtracted radial profiles drop to zero around $2^{\prime \prime}$, with most $(\sim 90 \%)$ of the flux contained within $1^{\prime \prime}$. In these cases, the observed radial profile is entirely consistent with the instrumental PSF. Figure 1a shows the radial profile for 0405-123, which is an example of the 16 quasi-stellar sources without diffuse emission, together with the residuals (ratios of the data to the PSF).

However, evidence for diffuse X-ray emission is present in the case of $0836+299$, the only FRI of the sample. As Fig. 1b clearly shows, this object has an excess of X-ray emission over the PSF and the background at 2-3 $\sigma$ level at $5^{\prime \prime}$ from the core. This is in keeping with the results of Worrall et al. (2001), who find that extended X-ray halos are a common property of lowpower radio galaxies.

We tried a fit to the radial profile with a King profile; however, the parameters of the model (the $\beta$ value and the core radius) are unconstrained. The intrinsic $0.4-5 \mathrm{keV}$ luminosity of the diffuse emission is $L_{0.4-5 \mathrm{keV}}=3.3 \times 10^{41} \mathrm{ergs} \mathrm{s}^{-1}$ (assuming a Raymond-Smith thermal plasma with temperature $k T=1 \mathrm{keV}$, solar abundances, and a Galactic column density), within the range measured for other FRIs $\left(L_{0.4-5 \mathrm{keV}} \sim\right.$ 2.6-4.2 $\times 10^{41} \mathrm{ergs} \mathrm{s}^{-1}$ ) (Worrall et al. 2001). Deeper ACIS observations are needed to confirm the presence of diffuse X-ray emission around the core of $0836+299$ and better quantify its properties.

We can determine limits for the detection of galaxy halos for all of the quasars of the sample, since each has a core radial profile that is consistent with a point source. Any small-scale
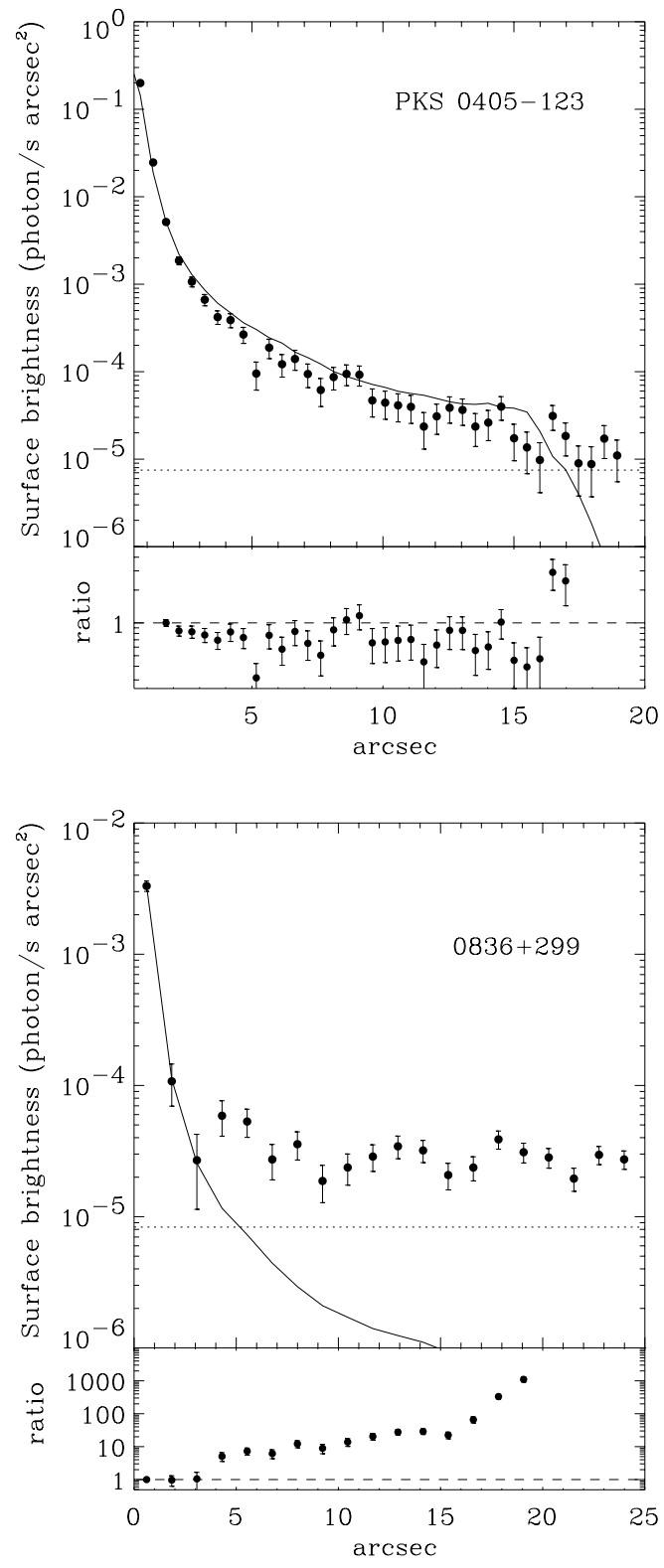

Fig. 1. (a), Top) Radial profile for 0405-123. The solid line is the instrumental profile for a point source. The dotted line is the background level. The plotted ratio of the surface brightness to the point-spread function shows that there is no extended emission present near this object, as there is none for the quasars of our sample. (b), Bottom) Radial profile for the nearby, low-power radio galaxy $0836+299$. In this case, there is evidence for diffuse X-ray emission on scales of a few kpc, consistent with other FRIs (Worrall et al. 2001).

diffuse X-ray emission, if present, is confined within 1", corresponding to $3.7 \mathrm{kpc}$ at $z=0.302$ and $5.5 \mathrm{kpc}$ at $z=1.956$, the two extreme redshifts of the quasar sample. In this case, while we can not resolve the diffuse component spatially, we could in principle detect a thermal emission component in the $\mathrm{X}$-ray spectrum of the core. Alternatively, any diffuse largerscale X-ray emission, if present on scales $>1^{\prime \prime}$, is too weak to be detected in our short Chandra exposures, and we can determine a detection threshold.

To assess our sensitivity to the thermal component, we performed a simulation with XSPEC assuming a power law 
Table 3. Timing analysis of quasar cores.

\begin{tabular}{cccc}
\hline \hline $\begin{array}{c}\text { Source } \\
(1)\end{array}$ & $\begin{array}{c}\chi_{\mathrm{r}}^{2} / \text { d.o.f. } \\
(2)\end{array}$ & $\begin{array}{c}P_{\chi^{2}} \\
(3)\end{array}$ & $\begin{array}{c}\text { Variable } \\
(4)\end{array}$ \\
\hline $0405-123$ & $1.064 / 19$ & 0.382 & $\mathrm{~N}$ \\
$0605-085$ & $0.261 / 19$ & 0.999 & $\mathrm{~N}$ \\
$0723+679$ & $0.881 / 20$ & 0.612 & $\mathrm{~N}$ \\
$0802+103$ & $0.458 / 18$ & 0.975 & $\mathrm{~N}$ \\
$0836+299$ & $0.436 / 17$ & 0.976 & $\mathrm{~N}$ \\
$0838+133$ & $0.650 / 24$ & 0.183 & $\mathrm{~N}$ \\
$1040+123$ & $0.999 / 23$ & 0.462 & $\mathrm{~N}$ \\
$1055+018$ & $0.534 / 20$ & 0.954 & $\mathrm{~N}$ \\
$1136-135$ & $0.676 / 19$ & 0.846 & $\mathrm{~N}$ \\
$1150+497$ & $0.863 / 20$ & 0.636 & $\mathrm{~N}$ \\
$1354+195$ & $0.638 / 19$ & 0.880 & $\mathrm{~N}$ \\
$1510-089$ & $2.626 / 21$ & $3.6 \mathrm{e}-9$ & $\mathrm{Y}$ \\
$1641+399$ & $1.005 / 19$ & 0.451 & $\mathrm{~N}$ \\
$1642+690$ & $0.771 / 18$ & 0.737 & $\mathrm{~N}$ \\
$1741+279$ & $0.440 / 19$ & 0.982 & $\mathrm{~N}$ \\
$1928+738$ & $1.405 / 18$ & 0.115 & $\mathrm{~N}$ \\
$2251+134$ & $0.570 / 20$ & 0.935 & $\mathrm{~N}$ \\
\hline
\end{tabular}

Columns explanation: 1 = Source IAU name; $2=$ Reduced $\chi^{2}$ and degrees of freedom (d.o.f.) for $500 \mathrm{~s}$ binning; $3=$ Probability of $\chi^{2}$ statistics; 4 = Flag indicating whether the source is variable $(\mathrm{Y})$, or not $(\mathrm{N})$ based on Col. (3).

model (associated with the active nucleus) with photon in$\operatorname{dex} \Gamma=1.66$ (average value from the sample) and flux $F_{2-10 \mathrm{keV}}=8 \times 10^{-13} \mathrm{ergs} \mathrm{cm}^{-2} \mathrm{~s}^{-1}$, at a median redshift of $z=0.6$. We added a Raymond-Smith thermal component with temperature $k T=1 \mathrm{keV}$ (typical of FRIs' halos), abundance fixed to 0.5 solar, and varied its normalization until the thermal component was no longer detected significantly, according to the F-test. We find that the latter occurs for a flux,

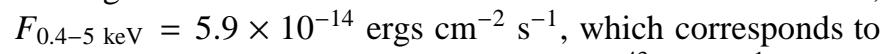
an intrinsic luminosity, $L_{0.4-5 \mathrm{keV}}=4.8 \times 10^{43} \mathrm{ergs} \mathrm{s}^{-1}$, greater than the typical halo luminosity measured for FRIs. A similar flux limit is obtained for $0838+133$, although it has a longer exposure, since its X-ray core is 4 times brighter than our average model.

To assess our sensitivity to diffuse emission on largerscales $\left(>1^{\prime \prime}, \sim 10 \mathrm{kpc}\right)$, we performed MARX simulations. We assumed a point source with an average core X-ray flux of $8 \times 10^{-13} \mathrm{ergs} \mathrm{cm}^{-2} \mathrm{~s}^{-1}$, at a median redshift of $z=0.6$ and a spectrum described by a power law with a photon index of 1.66. Following Worrall et al. (2001), the point source was described spatially as a Gaussian with width $\sigma=0.2$ to account for the aspect solution. For the diffuse X-ray emission we assumed a modified King profile for cluster emission, with the core radius fixed at $2^{\prime \prime}$ (or $10 \mathrm{kpc}$ intrinsic size for $z=0.6$ ), and the $\beta$ parameter fixed at 0.8 , and varied the $0.5-8 \mathrm{keV}$ flux. We find that diffuse $\mathrm{X}$-ray emission can be detected down to a surface brightness of $1 \times 10^{-14} \mathrm{ergs} \mathrm{cm}^{-2} \mathrm{~s}^{-1} \operatorname{arcsec}^{-2}$ for a $10 \mathrm{ks}$ exposure, corresponding to the luminosity, $L_{0.4-5} \mathrm{keV}=$ $8.5 \times 10^{43} \mathrm{ergs} \mathrm{s}^{-1}$.

We conclude that, with the exception of the nearby lowpower galaxy $0836+299$, no diffuse X-ray emission related to the host galaxy's halo or cluster was detected around the cores of the quasars in our sample, even in the case of $0838+133$, which was observed for a significantly longer exposure $(37 \mathrm{ks})$. Since our exposures were short ( $10 \mathrm{ks})$ and the detected nuclear emission is relatively strong, it is possible that the diffuse emission is just too weak to be detected against a very bright core in these relatively distant quasars. Our simulations show that any unresolved $\left(\$ 1^{\prime \prime}\right)$ diffuse emission on the scale of the galaxy halo would be fainter than $F_{0.4-5 \mathrm{keV}}=5.9 \times 10^{-14} \mathrm{ergs} \mathrm{cm}^{-2} \mathrm{~s}^{-1}$, while any largerscale diffuse emission would have a surface brightness $\lesssim 1 \times$ $10^{-14} \mathrm{erg} \mathrm{cm}^{-2} \mathrm{~s}^{-1} \operatorname{arcsec}^{-2}$.

\subsection{X-ray variability on short and long timescales}

Most (13/17) of the sources in this sample are classified as variable at optical and other wavelengths, and two of them are also known from previous studies to exhibit long- and short-term variations of the X-ray flux. The blazar 1510-089 showed a factor of $\sim 3.5$ variation in flux on a timescale $\sim 2$ years, between survey and pointed observations with ROSAT (Siebert et al. 1996). The blazar $1641+399$ showed a factor of $\sim 2$ variation also on a timescale $\sim 2$ years (Sambruna 1997). Here we examine whether short-term variability is also present within our Chandra exposures.

Table 3 reports the results of the $\chi^{2}$ test applied to the 500 s-bin Chandra light curves of the core, and the probability that the light curve is constant ( $\chi^{2}$ probability). No highamplitude variability is present based on this test.

Only 1510-089 has a large enough reduced $\chi^{2}$ value, $\chi_{\mathrm{r}}^{2}=2.63$, to indicate possible variability. Inspection of its light curve shows erratic excursions of the X-ray flux, with no apparent regular trend at the $500 \mathrm{~s}$ binning. For this reason the Kolmogorov-Smirnov (KS) test for a probability of constancy was applied to this object at a variety of binnings. The results of this test show that the probability of constancy for this object is $6.7 \%$ at binnings of 750,250 and $100 \mathrm{~s}$, but is $10 \%$ at a binning of $500 \mathrm{~s}$, the most erratic bin set. In other words, according to the KS test the probability of variability in the light curve ranges between $90 \%$ and $93.3 \%$. Figure 2 shows the light curve for this object binned at $750 \mathrm{~s}$. A change of the flux by a factor of 1.4 within $25 \mathrm{~min}$ is apparent.

We also investigated whether the flux variability in 1510-089 is energy-dependent. Light curves binned at $750 \mathrm{~s}$ were accumulated in a soft $(0.3-1 \mathrm{keV})$ and a hard $(2-8 \mathrm{keV})$ energy band, and the $\chi^{2}$ and KS tests were applied. At soft Xrays we find a $92 \%$ probability of variation in the light curve, and at hard X-rays we find a $96 \%$ probability of variation. We conclude that variability is present at both soft and hard X-rays in 1510-089.

In conclusion, none of the objects decisively demonstrated short-term, high-amplitude variability in our Chandra exposures. The only exception is $1510-089$ which showed a lowamplitude flux change within a timescale of $\sim 25 \mathrm{~min}$. We suggest long-exposure observations with Chandra or XMMNewton of this object for confirmation of this result. 


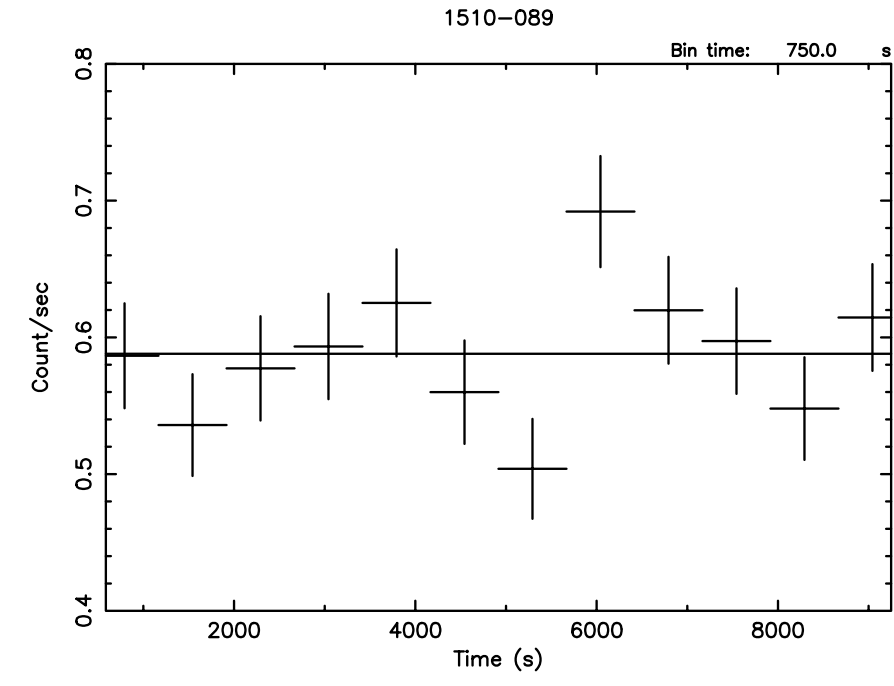

Fig. 2. Light curve of $1510-089$ binned at $750 \mathrm{~s}$ intervals. Lowamplitude flux changes on a timescale of $25 \mathrm{~min}$ are present.

We also checked for long-term X-ray variability by comparing the Chandra flux with previously published X-ray fluxes from other experiments. We decided to compare the monochromatic $1 \mathrm{keV}$ fluxes, because these are less sensitive to the shape of the X-ray continuum and are not based on the different waveband sensitivities of earlier experiments. The Chandra monochromatic flux at $1 \mathrm{keV}$ is reported in Table 7 for each object, while previous X-ray observations of the sources in our sample are listed in Appendix A. As a caveat it is worth noting that the X-ray fluxes from the pre-Chandra experiments used much larger extraction radii (of the order of arcminutes); however, as shown by our spatial analysis, the nucleus is the dominant source of X-ray emission in these regions. In Table 7 , five objects are found to be long-term X-ray variables: $0405-123$ shows a factor of $\sim 1.5$ decrease in 9 years (Sambruna 1997); 1055+018 a factor of $\sim 1.5$ increase in 8 years (Siebert et al. 1998); 1354+195 a factor of $\sim 3$ decrease in $\sim 20$ years (Biermann et al. 1987); 1510-089 a factor of $\sim 1.5$ decrease in $\sim 8$ years (Siebert et al. 1998; Singh et al. 1990); and $1928+738$ a factor of $\sim 2$ decrease in 10 years (Lawson et al. 1992).

\subsection{X-ray continuum emission}

Quasar cores: The results of the spectral fits with a single power law plus fixed Galactic absorption are reported in Table 4, including the observed $2-10 \mathrm{keV}$ fluxes, and the intrinsic (absorption-corrected) $2-10 \mathrm{keV}$ luminosities. All fitted parameters are listed with their $90 \%$ confidence errors, $\left(\Delta \chi^{2}=2.7\right.$ for one parameter of interest).

In general, a single power law plus Galactic absorption provides an acceptable description of the $0.5-8 \mathrm{keV}$ continua, as judged by inspection of the residuals and from the values of $\chi_{\mathrm{r}}^{2}$ from Table 4. For two sources $(0838+133$ and $1040+123)$, this model provides a large $\chi_{\mathrm{r}}^{2} \sim 1.26$; inspection of the residuals shows the presence of random fluctuations indicating a noisy spectrum. Complex models do not improve the fits of these two objects.
Table 4. Single power law fits for the X-ray continua ${ }^{a}$.

\begin{tabular}{ccccc}
\hline \hline $\begin{array}{c}\text { Source } \\
(1)\end{array}$ & $\begin{array}{c}\Gamma \\
(2)\end{array}$ & $\begin{array}{c}\chi_{\mathrm{r}}^{2} / \text { d.o.f. } \\
(3)\end{array}$ & $\begin{array}{c}F_{2-10 \mathrm{keV}} \\
(4)\end{array}$ & $\begin{array}{c}L_{2-10 \mathrm{keV}} \\
(5)\end{array}$ \\
\hline $0405-123$ & $1.90 \pm 0.05$ & $1.32 / 152$ & $4.8 \times 10^{-12}$ & $2.7 \times 10^{45}$ \\
$0605-085$ & $1.66 \pm 0.09$ & $1.03 / 55$ & $1.1 \times 10^{-12}$ & $1.2 \times 10^{45}$ \\
$0723+679$ & $1.76 \pm 0.08$ & $0.99 / 71$ & $9.1 \times 10^{-13}$ & $9.4 \times 10^{44}$ \\
$0802+103$ & $1.88 \pm 0.23$ & $0.60 / 9$ & $1.2 \times 10^{-13}$ & $4.4 \times 10^{44}$ \\
$0838+133$ & $1.35 \pm 0.06$ & $1.25 / 104$ & $1.2 \times 10^{-12}$ & $7.6 \times 10^{44}$ \\
$1040+123$ & $1.69 \pm 0.07$ & $1.26 / 76$ & $9.7 \times 10^{-13}$ & $1.3 \times 10^{45}$ \\
$1055+018$ & $1.67 \pm 0.07$ & $1.12 / 86$ & $2.7 \times 10^{-12}$ & $3.0 \times 10^{45}$ \\
$1136-135$ & $1.76 \pm 0.09$ & $0.99 / 44$ & $1.2 \times 10^{-12}$ & $1.8 \times 10^{45}$ \\
$1150+497$ & $1.86 \pm 0.06$ & $1.22 / 86$ & $2.2 \times 10^{-12}$ & $4.3 \times 10^{44}$ \\
$1354+195$ & $1.56 \pm 0.07$ & $1.06 / 75$ & $2.6 \times 10^{-12}$ & $6.4 \times 10^{45}$ \\
$1510-089$ & $1.40 \pm 0.06$ & $1.31 / 102$ & $5.1 \times 10^{-12}$ & $1.1 \times 10^{45}$ \\
$1641+399$ & $1.71 \pm 0.07$ & $1.18 / 82$ & $2.6 \times 10^{-12}$ & $1.5 \times 10^{45}$ \\
$1642+690$ & $1.77 \pm 0.10$ & $0.96 / 51$ & $8.0 \times 10^{-13}$ & $6.8 \times 10^{44}$ \\
$1741+279$ & $2.05 \pm 0.13$ & $0.98 / 36$ & $8.0 \times 10^{-13}$ & $2.0 \times 10^{44}$ \\
$1928+738$ & $1.88 \pm 0.07$ & $1.01 / 98$ & $3.3 \times 10^{-12}$ & $5.4 \times 10^{44}$ \\
$2251+134$ & $1.82 \pm 0.08$ & $1.13 / 65$ & $7.8 \times 10^{-13}$ & $5.6 \times 10^{44}$ \\
\hline
\end{tabular}

Columns explanation: 1 = Source IAU name; $2=$ Photon index and $90 \%$ uncertainties $\left(\Delta \chi^{2}=2.7\right) ; 3=$ Reduced $\chi^{2}$ value and degrees of freedom (d.o.f.); 4 = Absorbed flux in $2-10 \mathrm{keV}$ in ergs $\mathrm{cm}^{-2} \mathrm{~s}^{-1}$; 5 = Intrinsic X-ray luminosity in $2-10 \mathrm{keV}$ assuming redshift (Table 1) and cosmology $\left(H_{0}=75 \frac{\mathrm{km} \mathrm{s}^{-1}}{\mathrm{Mpc}}, q_{0}=0.5\right)$ in ergs s $\mathrm{s}^{-1}$.

Notes: $a=$ Radio galaxy $0836+299$ did not have an acceptable single power law fit (Sect. 3.3) and is listed in Table 5.

The single power law plus Galactic $N_{\mathrm{H}}$ model is not an adequate description of the X-ray continuum in 6 quasars $(0405-123,1055+018,1150+497,1354+195,1510-089$, and $1641+399)$. These sources show complex residuals which require additional spectral components at low energies. In all six cases, excess flux at soft X-rays $(\sim 1 \mathrm{keV})$ is present over the extrapolation of the power law model. We modeled the soft excess flux in terms of three possible alternative models: a) thermal bremmstrahlung, b) blackbody, and c) broken power law. In all cases, a broken power law with a steep index below $\sim 1.6 \mathrm{keV}$ is the preferred model based on the $\chi_{\mathrm{r}}^{2}$. These best-fit models are reported in Table 5.

No excess absorption was detected in the quasars of our sample, including the quasar $0838+133$, for which Brunetti et al. (2002) found excess column density over Galactic. After making a correction for the molecular contamination of ACIS, the column density we measured in $0838+133$ was consistent with Galactic values.

The low-power radio galaxy 0836+299: In this source, inspection of the spectrum shows a marked deficit of counts below $2 \mathrm{keV}$, indicating large excess absorption over the Galactic value. A formal fit with a single power law plus free absorption converges to a negative value of the photon index and zero column density, which simply parameterizes excess absorption at low energies, and is not physically acceptable. We fitted the spectrum of $0836+299$ with a power law plus Galactic $N_{\mathrm{H}}$ model, adding an extra column density at the redshift of the source in the assumption that the excess absorption is intrinsic (this is a nearby source). Because of the limited number of 
Table 5. More complex continuum fits.

\begin{tabular}{|c|c|c|c|c|c|}
\hline $\begin{array}{l}\text { Source } \\
(1)\end{array}$ & $\begin{array}{l}\text { Best-fit model and parameters } \\
\text { (2) }\end{array}$ & $\begin{array}{c}\chi_{\mathrm{r}}^{2} / \text { d.o.f. } \\
\text { (3) }\end{array}$ & $\begin{array}{c}\Delta \chi^{2} / P_{F} \\
(4)\end{array}$ & $\begin{array}{l}F_{2-10 \mathrm{keV}} \\
(5)\end{array}$ & $\begin{array}{l}L_{2-10 \mathrm{keV}} \\
\quad(6)\end{array}$ \\
\hline $0405-123$ & $\begin{array}{l}\Gamma_{\text {SOFT }}=2.37_{-0.15}^{+0.18} \\
\left(E_{0}=1.07_{-0.11}^{+0.13} \mathrm{keV}, \Gamma_{\text {HARD }}=1.74_{-0.08}^{+0.07}\right)\end{array}$ & $1.14 / 150$ & $31.7 / 99 \%$ & $5.4 \times 10^{-12}$ & $2.9 \times 10^{45}$ \\
\hline $0836+299$ & $\begin{array}{l}\text { power law }(\Gamma=1.69, \text { fixed }) \text { and } \\
\text { absorption }\left(N_{\mathrm{H}}=4.8_{-1.4}^{+1.9} \times 10^{23} \mathrm{~cm}^{-2}\right)\end{array}$ & $0.83 / 7$ & $\cdots$ & $1.1 \times 10^{-12}$ & $3.4 \times 10^{43}$ \\
\hline $1055+018$ & $\begin{array}{l}\Gamma_{\text {SOFT }}=2.11_{-0.26}^{+0.27} \\
\left(E_{0}=1.05_{-0.17}^{+0.34} \mathrm{keV}, \Gamma_{\text {HARD }}=1.54_{-0.09}^{+0.08}\right)\end{array}$ & $1.02 / 84$ & $11.4 / 99 \%$ & $3.0 \times 10^{-12}$ & $3.1 \times 10^{45}$ \\
\hline $1150+497$ & $\begin{array}{l}\Gamma_{\text {SOFT }}=2.14_{-0.13}^{+0.16} \\
\left(E_{0}=1.37_{-0.25}^{+0.60} \mathrm{keV}, \Gamma_{\text {HARD }}=1.74_{-0.12}^{+0.11}\right)\end{array}$ & $1.07 / 83$ & $16.3 / 99 \%$ & $2.4 \times 10^{-12}$ & $4.6 \times 10^{44}$ \\
\hline $1354+195$ & $\begin{array}{l}\Gamma_{\text {SOFT }}=1.88_{-0.28}^{+0.37} \\
\left(E_{0}=1.09_{-0.20}^{+0.32} \mathrm{keV}, \Gamma_{\text {HARD }}=1.39_{-0.09}^{+0.10}\right)\end{array}$ & $0.83 / 73$ & $19.4 / 99 \%$ & $2.9 \times 10^{-12}$ & $6.8 \times 10^{45}$ \\
\hline $1510-089$ & $\begin{array}{l}\Gamma_{\text {SOFT }}=1.68_{-0.16}^{+0.24} \\
\left(E_{0}=1.57_{-0.44}^{+0.53} \mathrm{keV}, \Gamma_{\text {HARD }}=1.22_{-0.12}^{+0.16}\right)\end{array}$ & $1.21 / 100$ & $12.3 / 99 \%$ & $5.7 \times 10^{-12}$ & $1.2 \times 10^{45}$ \\
\hline $1641+399$ & $\begin{array}{l}\Gamma_{\text {SOFT }}=2.05_{-0.13}^{+0.14} \\
\left(E_{0}=1.59_{-0.22}^{+0.31} \mathrm{keV}, \Gamma_{\text {HARD }}=1.37_{-0.15}^{+0.12}\right)\end{array}$ & $0.88 / 80$ & $26.6 / 99 \%$ & $3.3 \times 10^{-12}$ & $1.7 \times 10^{45}$ \\
\hline
\end{tabular}

Columns explanation: 1 = Source IAU name; 2 = Best-fit models: for all objects, except $0836+299$, a broken power law is used. In this model, $E_{0}$ is the break energy, $\Gamma_{\mathrm{SOFT}}$ is the photon index below $E_{0}, \Gamma_{\mathrm{HARD}}$ is the photon index above $E_{0} ; 3=$ Reduced $\chi^{2}$ and degrees of freedom

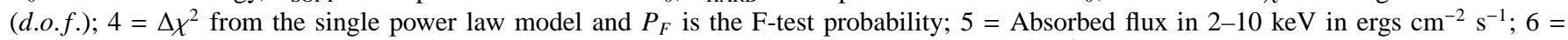
Intrinsic X-ray luminosity in 2-10 keV assuming redshift (Table 1$)$ and cosmology $\left(H_{0}=75 \frac{\mathrm{km} \mathrm{s}^{-1}}{\mathrm{Mpc}}, q_{0}=0.5\right)$ in ergs s . $^{-1}$.

counts, the photon index of the power law was fixed to the average found for the other lobe-dominated sources $(\Gamma=1.69$; see below). We found a significant intrinsic column density, $N_{\mathrm{H}} \sim 5 \times 10^{23} \mathrm{~cm}^{-2}$ (Table 5). The fitted column does not vary significantly if the photon index is fixed to the extrema of the range observed for this sample.

\subsection{Fe K-shell emission}

As discussed above (Sect. 1), Fe K-shell emission in higherluminosity sources, both radio-quiet and radio-loud, is not well established. Interestingly, we find evidence for possible Fe K line emission in two sources of our sample, $0723+679$ and $1150+497$, and possibly in a third object, $1642+690$.

Inspection of the residuals of the power law models shows excess emission around $3.5 \mathrm{keV}, 4.8 \mathrm{keV}$, and $3.7 \mathrm{keV}$ for $0723+679,1150+497$, and $1642+690$, respectively. These energies are consistent with the redshifted $\mathrm{Fe} \mathrm{K} \alpha$ line energy, 6.4 $\mathrm{keV}$. To model the line, a Gaussian component was added to the power law models reported in Table 4 for each of the three sources. The significance of the $\chi^{2}$ improvement is $97 \%$ for $0723+679$ and $1150+497$, and $94 \%$ for $1642+690$. In Fig. 3 we show the best-fit model and data for $0723+679$ and $1150+497$, for which the line detections are more significant. Also shown in Fig. 3 are the confidence contours for the line flux versus line width in both sources. The Fe $\mathrm{K} \alpha$ line is detected at the $\gtrsim 90 \%$ confidence level and is always unresolved. Since the line is unresolved, we fixed the line width at $\sigma=0.05 \mathrm{keV}$, lower than the S3 resolution (from Fig. 6.6 from the Chandra POG, $\sigma \sim 0.2 \mathrm{keV}$ at $6 \mathrm{keV}$ ), and performed the fit - leaving the Gaussian center energy and normalization free to vary. The fitted parameters (energy and $E W$, both in the rest-frame) for a narrow line are listed in Table 6, with their $90 \%$ confidence errors. The fitted rest-frame emission line energies are consistent with the $\mathrm{K} \alpha$ fluorescent line from cold Fe. The fitted, rest-frame $E W \mathrm{~s}$ are $164_{-138}^{+455}, 296_{-203}^{+538}$, and $188_{-54}^{+748} \mathrm{eV}$ for $0723+679,1150+497$, and $1642+690$, respectively. Deeper observations of these targets with Chandra and/or XMMNewton are needed to confirm the line and study its profile.

For the remaining 14 sources, we determined an upper limit to the $\mathrm{Fe} \mathrm{K} \alpha$ line $E W \mathrm{~s}$. We fitted the spectra adding a Gaussian component at an energy consistent with the redshifted energy of the $\mathrm{Fe} \mathrm{K} \alpha$ line and assumed an unresolved line $(\sigma=0.05 \mathrm{keV})$. The $90 \%$ confidence upper limits on the line $E W \mathrm{~s}$ (in the source's rest-frame) are reported in Table 6. For one source $(0836+299)$ the data included too few counts from the hard X-ray spectrum to determine an upper limit for the $\mathrm{Fe} \mathrm{K} \alpha$ emission line.

\section{Summary and discussion}

We presented the X-ray properties of the cores of 16 radioloud quasars and one low-power radio galaxy observed with Chandra in short ( $\sim 10 \mathrm{ks})$ exposures. The targets were selected for their large-scale radio properties, so the Chandra observations were optimized for the detection of radio jets at X-rays. Thus, the sample is not statistical as far as the core and extended properties are concerned.

The Chandra resolution allows us to separate the nuclear $\mathrm{X}$-ray emission from the extended (kpc-scale) jet for the first time. In general, we find that the cores dominate the total $\mathrm{X}$ ray emission from the source, with a large ratio of core-to-jet counts (Table 2, Col. 6). The only exceptions are the lobedominated sources $0802+103$ and $0836+299$, where the core $\mathrm{X}$-ray emission is comparable to and smaller than the jet, respectively. However, it is necessary to stress that the $2^{\prime \prime}$ aperture used for the core spectrum extraction corresponds to a region of intrinsic size $\sim 10 \mathrm{kpc}$ at a median redshift of $z=0.6$ 

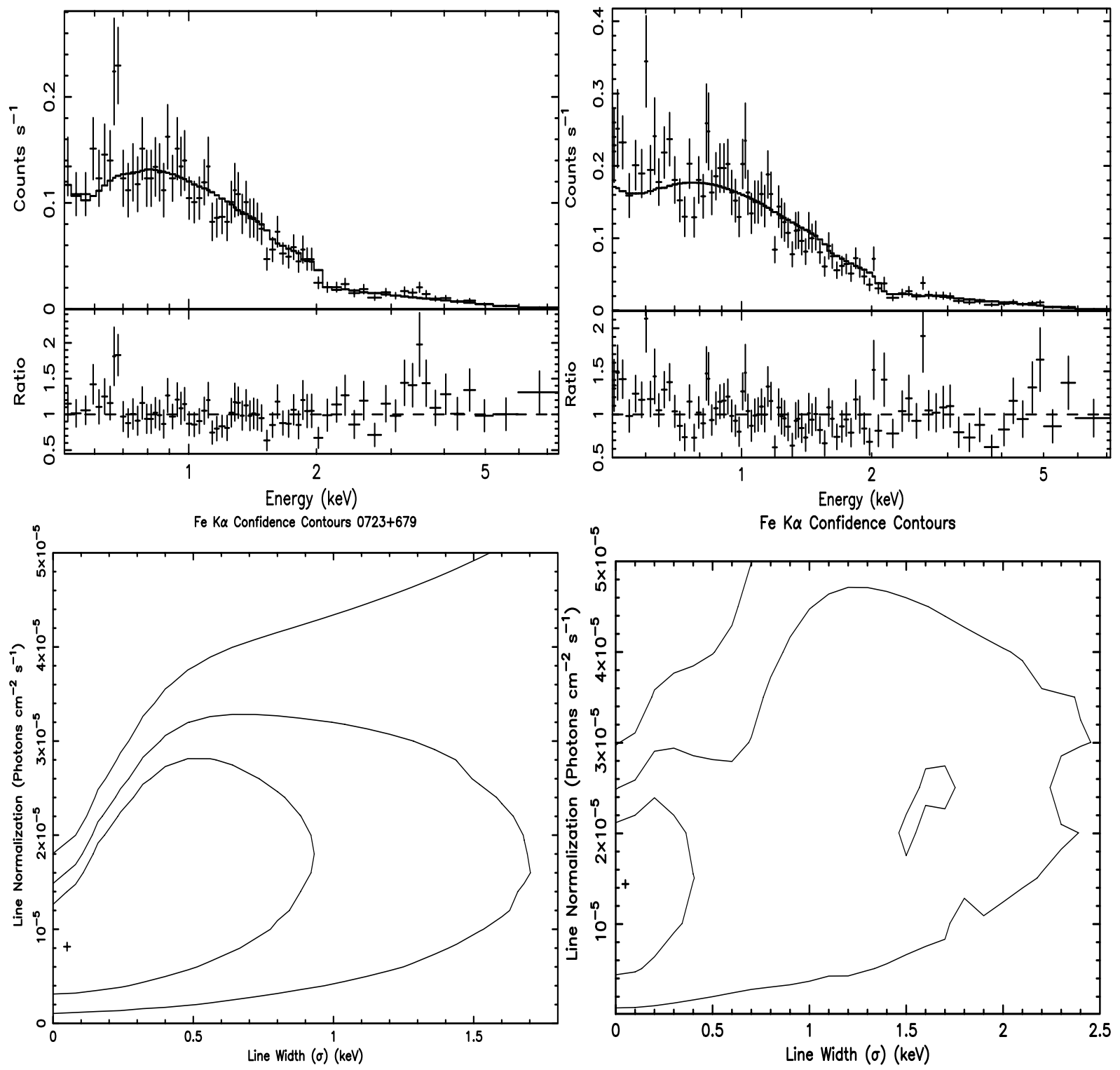

Fig. 3. Results of complex spectral fits of the quasars $0723+679$ (left) and 1150+497 (right). Top panels: observed ACIS-S spectra and residuals of a single power law plus Galactic absorption model. An Fe K line is present at observed energies 3.5 and $4.8 \mathrm{keV}$. Bottom panels: confidence contours at 68\%,90\%,99\% confidence for the Gaussian line normalization versus width. In both sources, the line is detected at $\gtrsim 90 \%$ confidence and is always unresolved.

( $2 \mathrm{kpc}$ in the near source 0836+299). So, the "core" X-ray emission measured with Chandra is likely contaminated by the inner regions of the jet. Indeed, the ratios of the jet-tocounterjet flux from radio observations (Cheung et al. 2003) indicate that most of the jets in these sources are closely aligned to our line of sight. Worrall et al. (2001) also found that the Xray jet is a substantial fraction of the total X-ray output in the B2 FRI sample.

\subsection{Nuclear X-ray emission}

The X-ray continua can be described by a power law, with further spectral complexities (cold absorption in excess of Galactic, soft excess, Fe K line) in 9/17 sources. Using the photon indices from the best-fit models in Tables 4 and 5, we calculated the average slope of the $2-10 \mathrm{keV}$ continuum. In the cases with a soft excess (Table 5), we used the photon index above the break energy. The average photon index for the whole sample is $\left\langle\Gamma_{\text {sample }}\right\rangle=1.66$ with standard deviation $\sigma_{\text {sample }}=0.23$.

It is interesting to compare the average X-ray slope from our sample $\left(\left\langle\Gamma_{\text {sample }}\right\rangle=1.66, \sigma_{\text {sample }}=0.23\right)$, with the slope derived for radio sources (BLRGs and quasars) with jets that are known to be oriented at large angles to the line of sight (Hasenkopf et al. 2002; Eracleous \& Halpern 1998). These sources have inclination angles larger than $15^{\circ}$ (Eracleous \& Halpern 1998), or are classical radio doubles where the line of sight is not too close to the jet (Hasenkopf et al. 2002). From 
Table 6. Fe K-shell emission ${ }^{a}$.

\begin{tabular}{ccccc}
\hline \hline Source & $E_{\ell}$ & $E W$ & $\Delta \chi^{2}$ & $P_{F}$ \\
$(1)$ & $(2)$ & $(3)$ & $(4)$ & $(5)$ \\
\hline \multicolumn{5}{c}{ Detections } \\
$0723+679$ & $6.4_{-0.1}^{+0.1}$ & $164_{-138}^{+455}$ & 6.1 & $97 \%$ \\
$1150+497$ & $6.4_{-0.1}^{+0.6}$ & $296_{-203}^{+538}$ & 5.0 & $97 \%$ \\
$1642+690$ & $6.4_{-0.7}^{+0.8}$ & $188_{-54}^{+748}$ & 3.4 & $94 \%$ \\
\multicolumn{5}{c}{ Upper Limits ${ }^{b, c}$} \\
$0405-123$ & 6.4 & 294 & $\ldots$ & $\ldots$ \\
$0605-085$ & 6.4 & 484 & $\ldots$ & $\ldots$ \\
$0802+103$ & 6.4 & 865 & $\ldots$ & $\ldots$ \\
$0838+133$ & 6.4 & 953 & $\ldots$ & $\ldots$ \\
$1040+123$ & 6.4 & 528 & $\ldots$ & $\ldots$ \\
$1055+018$ & 6.4 & 324 & $\ldots$ & $\ldots$ \\
$1136-135$ & 6.4 & 992 & $\ldots$ & $\ldots$ \\
$1354+195$ & 6.4 & 504 & $\ldots$ & $\ldots$ \\
$1510-089$ & 6.4 & 434 & $\ldots$ & $\ldots$ \\
$1641+399$ & 6.4 & 493 & $\ldots$ & $\ldots$ \\
$1741+279$ & 6.4 & 908 & $\ldots$ & $\ldots$ \\
$1928+738$ & 6.4 & 829 & $\ldots$ & $\ldots$ \\
$2251+134$ & 6.4 & 751 & $\ldots$ & $\ldots$ \\
\hline
\end{tabular}

Columns explanation: 1 = Source IAU name; 2 = Rest-frame line energy in $\mathrm{keV}$, with line width fixed at $\sigma=0.05 \mathrm{keV} ; 3=$ Restframe Equivalent width $(E W)$ in $\mathrm{eV} ; 4=\Delta \chi^{2}$ from the best-fit model (Tables 4 or 5 ); 5 = F-test probability.

Notes: $a=$ In all cases, an unresolved line was assumed and the Gaussian width was fixed to $\sigma=0.05 \mathrm{keV}$, reported errors are $90 \%$ confidence errors; $b=$ No upper limit is listed for $0836+299$ (see text Sect. 3.4); $c=$ Line Energy is fixed at $E_{\ell}=6.4 \mathrm{keV}$.

ASCA, RXTE, and BeppoSAX observations of these sources, an average photon index of $\langle\Gamma\rangle=1.83$ and dispersion 0.04 is obtained in a range of intrinsic X-ray luminosities similar to our sources. The average slope for the more misaligned sources is steeper than for our sources (within large dispersions), supporting the idea that contamination from the unresolved, inner parts of a jet is present in our sample. Indeed, the average photon index for our quasars is consistent with the average X-ray photon index measured with BeppoSAX for a sample of gamma-ray bright blazars (Tavecchio et al. 2000), where beaming is important. The average slope for our sample is also flatter than for radio-quiet quasars, where $\left\langle\Gamma_{\mathrm{RQQ}}\right\rangle=1.89$ and dispersion 0.05 (Reeves \& Turner 2000).

Previous studies of radio-loud quasars with ROSAT (Elvis et al. 1994) indicated the presence of excess cold absorption over the Galactic value in high- $z$ sources. At odds with this result, we do not find evidence for excess $\mathrm{X}$-ray absorption in our two $z>1$ quasars, $0802+103(z=1.96)$ and $1040+123$ $(z=1.03)$. Interestingly, we do find evidence for excess X-ray absorption in the closest source of the sample, 0836+299 (see Sect. 4.3).

In 6/17 sources, excess X-ray flux at soft energies is detected. Our best-fit parameterization is a broken power law, with a steep component below $1 \mathrm{keV}$. Inspection of the Spectral Energy Distributions of these soft excess quasars using data compiled by us shows that the best sampled sources of this group (0405-123, 1354+195, 1510-089, and 1641+399) exhibit a Blue Bump (BB) in the optical part of the spectrum. It is likely that the soft X-ray excess detected in our ACIS-S spectra is related to the high-energy tail of the $\mathrm{BB}$, for which a possible origin is thermal emission from an optically thick accretion disk (e.g., Czerny \& Elvis 1987). This would indicate the presence of a strong isotropic radiation field in these sources which is important for models of their high-energy emission (Tavecchio et al. 2000). Alternatively, the soft X-ray excess could be related to non-thermal (synchrotron) emission from the inner jet. In this case, one could expect to observe significant short-term flux variability as a result of synchrotron radiative losses. The possible detection of flux changes on timescales of $\sim 25 \mathrm{~min}$ in 1510-089, if confirmed, would indicate the presence of non-thermal emission at soft X-rays in this source. Future XMM-Newton observations are needed to confirm and better study the soft X-ray excess in the quasars of our sample.

We detect an $\mathrm{Fe} \mathrm{K} \alpha$ emission line in two sources $(0723+679$ and $1150+497)$ and possibly in a third $(1642+690)$ source. The detection of such a line is very interesting di per $s e$, because the presence of Fe K-shell emission from highluminosity AGNs in general, and from radio-loud sources in particular, is still not established (see Sect. 1). While it is important to confirm these lines and study their profile with higher-quality X-ray spectra, we note that their measured restframe $E W \mathrm{~s}$ (Table 6) are consistent with the $E W \mathrm{~s}$ of the other radio-sources with a double-lobe radio morphology and similar intrinsic X-ray luminosity recently studied by $A S C A$ (Hasenkopf et al. 2002). Usually, dilution of the X-ray continuum by a beamed jet component would weaken the strength of the Fe K lines in radio-loud quasars. This could suggest weaker beaming in 0723+679 (indeed a SSRQ) and in 1150+497 and 1642+690 (both FSRQs).

Although beaming is present in all the quasars of our sample as indicated by their broad-band X-ray continuum properties, we conclude that beaming is likely to affect the individual sources to a different degree.

\subsection{Core- and lobe-dominated sources}

Splitting the sample into core- and lobe-dominated sources based on Table 1 (Col. 7), we find no difference in the average photon index for the two subgroups: for the 11 coredominated quasars, $\left\langle\Gamma_{\mathrm{cd}}\right\rangle=1.65$ and $\sigma_{\mathrm{cd}}=0.25$, and for the five lobe-dominated sources $\left\langle\Gamma_{\mathrm{ld}}\right\rangle=1.69$ with $\sigma_{\mathrm{ld}}=0.20$. If we exclude the unusually flat source $0838+133$ (the only lobedominated source with strong core pileup), the range of the photon index for the lobe-dominated sources is greatly constrained: $\left\langle\Gamma_{\mathrm{ld}}\right\rangle=1.77$ and $\sigma_{\mathrm{ld}}=0.08$.

Figure 4 shows the plot of the X-ray photon index from Table 4 or 5 versus the ratio of core-to-extended flux $R_{i}$ from Table 1. Our measurements of the extended radio flux included contributions from the kpc-scale jets, which are presumably still beamed to some degree, so our values of $R_{i}$ do not follow the usual convention (Orr \& Browne 1982). Nevertheless our derived values are consistent with values from the literature, 


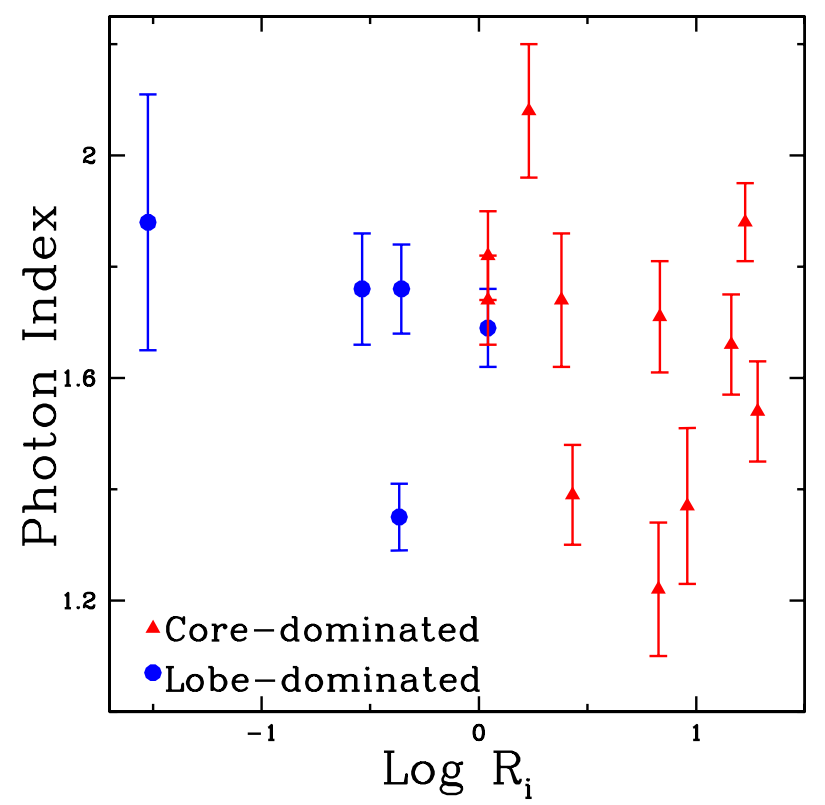

Fig. 4. Plot of the best-fit photon index of the X-ray continuum versus the core-dominance parameter $R_{i}$ for the 16 quasar sample. There is no trend for the whole quasar sample, nor within the subgroups of coreand lobe-dominated sources. The measured X-ray continuum slopes are similar for both subgroups.

where available (Cheung et al. 2003) and can be used to gauge the relative importance of beaming in these objects. There is neither any trend between the slope of the X-ray photon index and $R_{i}$ for the whole sample, nor for individual subgroups. The distribution of slopes for the core-dominated quasars overlaps with the distribution for lobe-dominated ones, and extends to flatter slopes; $4 / 11$ core-dominated sources have slopes $\Gamma \lesssim 1.6$, while $4 / 5$ lobe-dominated are steeper than this value.

We conclude that no difference in X-ray spectral slope is present between the core- and lobe-dominated subgroups of our sample. This result is not unexpected, as all the quasars including the SSRQs have one-sided radio jets and beaming is important in all cases. Larger samples of both core- and lobedominated quasars with optimal selection criteria are needed to verify these X-ray spectral properties.

We also compared the broad-band spectral distributions of both quasar subclasses. Broad-band spectral indices between radio $(5 \mathrm{GHz})$ and optical ( $V$ band), $\alpha_{\text {ro }}$, between optical and $\mathrm{X}$-rays $(1 \mathrm{keV}), \alpha_{\mathrm{ox}}$, and between radio and X-rays, $\alpha_{\mathrm{rx}}$, were calculated using published values and the Chandra fluxes from this work. Figure 5 shows the plot of the broad-band indices, $\alpha_{\text {ro }}$ versus $\alpha_{\text {ox }}$, while the broad-band monochromatic values and the plotted indices are listed in Table 7 . We see no marked difference between core- and lobe-dominated objects from the broad-band spectra. Overall, the quasars of our sample occupy a region of steeper radio-to-optical flux ratios than usually found for blazars (e.g., Donato et al. 2001), possibly as a result of their selection criteria based on prominent radio emission. The outlier in Fig. 5 is the FRI source 0836+299 (see below), which stands out for its flatter $\alpha_{\text {ox }}$ than the rest of the sample.

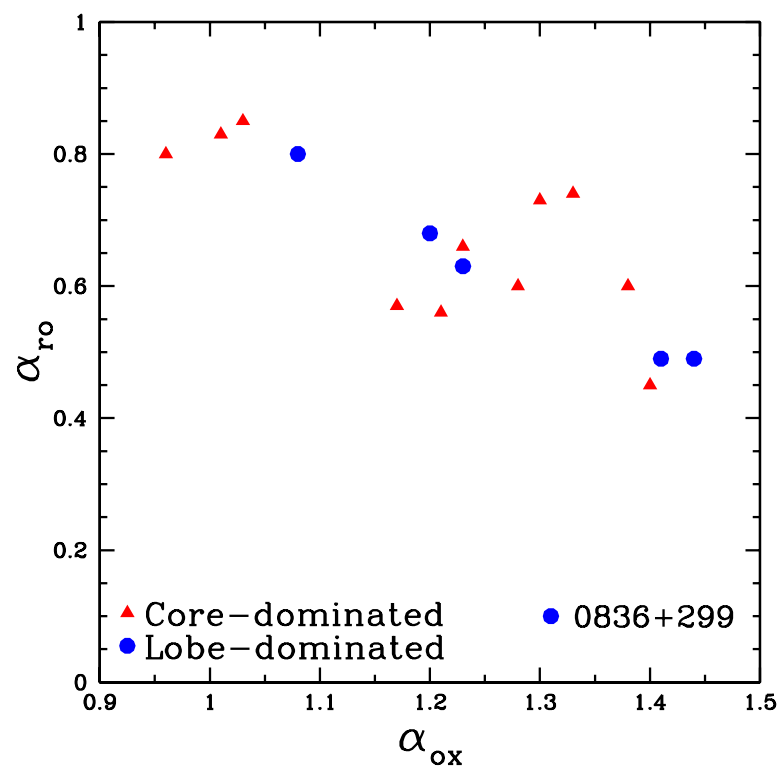

Fig. 5. Broad-band spectral properties of the quasars and of the radio galaxy of our sample. There is no difference between the core- and lobe-dominated subgroups. The FRI galaxy 0836+299 sticks out for its flat optical-to-X-ray flux ratio.

Table 7. Core broad-band emission.

\begin{tabular}{ccccccc}
\hline \hline $\begin{array}{c}\text { Source } \\
(1)\end{array}$ & $\begin{array}{c}F_{5 \mathrm{GHz}} \\
(2)\end{array}$ & $\begin{array}{c}F_{5500 \AA}(3) \\
F_{1 \mathrm{keV}} \\
(4)\end{array}$ & $\begin{array}{c}\alpha_{\text {ro }} \\
(5)\end{array}$ & $\begin{array}{c}\alpha_{\mathrm{ox}} \\
(6)\end{array}$ & $\begin{array}{c}\alpha_{\mathrm{rx}} \\
(7)\end{array}$ \\
\hline $0405-123$ & 0.961 & 5.6 & 1.01 & 0.45 & 1.40 & 0.78 \\
$0605-085$ & 2.252 & 0.50 & 0.17 & 0.73 & 1.30 & 0.93 \\
$0723+679$ & 0.423 & 0.31 & 0.16 & 0.63 & 1.23 & 0.84 \\
$0802+103$ & 0.040 & 0.18 & 0.03 & 0.47 & 1.41 & 0.80 \\
$0836+299$ & 0.008 & 2.48 & 0.80 & 0.10 & 1.31 & 0.52 \\
$0838+133$ & 0.483 & 0.19 & 0.12 & 0.68 & 1.20 & 0.86 \\
$1040+123$ & 1.163 & 0.12 & 0.16 & 0.80 & 1.08 & 0.89 \\
$1055+018$ & 3.256 & 0.22 & 0.44 & 0.83 & 1.01 & 0.89 \\
$1136-135$ & 0.463 & 1.67 & 0.23 & 0.49 & 1.44 & 0.82 \\
$1150+497$ & 0.443 & 0.61 & 0.47 & 0.57 & 1.17 & 0.78 \\
$1354+195$ & 1.755 & 1.69 & 0.34 & 0.60 & 1.38 & 0.87 \\
$1510-089$ & 1.482 & 1.48 & 0.56 & 0.60 & 1.28 & 0.84 \\
$1641+399$ & 8.133 & 1.59 & 0.44 & 0.74 & 1.33 & 0.95 \\
$1642+690$ & 1.403 & 0.08 & 0.14 & 0.85 & 1.03 & 0.91 \\
$1741+279$ & 0.204 & 0.31 & 0.18 & 0.56 & 1.21 & 0.79 \\
$1928+738$ & 2.991 & 1.46 & 0.73 & 0.66 & 1.23 & 0.86 \\
$2251+134$ & 0.595 & 0.06 & 0.16 & 0.80 & 0.96 & 0.85 \\
\hline
\end{tabular}

Columns explanation: 1 = Source IAU name; $2=5 \mathrm{GHz}$ flux in Jy (Cheung et al. 2003); 3 = Dereddened $5500 \AA$ flux in mJy; $4=1 \mathrm{keV}$ Chandra flux in $\mu \mathrm{Jy}$ from best-fit in Table 4 or $5 ; 5-7=$ Spectral Indices for radio-optical, optical-X-ray, and radio-X-ray $\left(F_{v} \propto v^{-\alpha}\right)$.

\subsection{The low-power radio galaxy $0836+299$}

The nearby FRI radio galaxy $0836+299$ has interesting properties which distinguish it from the other quasars in our sample. This is the only source with evidence for diffuse X-ray emission on the galaxy's halo scale, with X-ray luminosities consistent with other FRIs (Worrall et al. 2001).

Interestingly, we find evidence for excess absorption from the core, with column density $N_{\mathrm{H}} \sim 5 \times 10^{23} \mathrm{~cm}^{-2}$ (Table 5). 
Table 8. Earlier spectral fits for X-ray continua.

\begin{tabular}{|c|c|c|c|c|c|c|c|}
\hline $\begin{array}{c}\text { Source } \\
(1)\end{array}$ & $\begin{array}{l}N_{\mathrm{H}} \\
(2)\end{array}$ & $\begin{array}{c}\text { Photon index } \\
\text { (3) }\end{array}$ & $\begin{array}{l}\text { Other parameters } \\
\text { (4) }\end{array}$ & $\begin{array}{c}\chi_{\mathrm{r}}^{2} / d . o . f . \\
(5)\end{array}$ & $\begin{array}{c}F_{1 \mathrm{keV}} \\
(6)\end{array}$ & $\begin{array}{c}\text { Instrument/band } \\
\text { (7) }\end{array}$ & $\begin{array}{c}\text { References } \\
\text { (8) }\end{array}$ \\
\hline \multirow[t]{3}{*}{$0405-123$} & 7.20 & $\Gamma=1.76_{-0.10}^{+0.09}$ & w & $1.00 / 160$ & 0.90 & $A S C A(2-10)$ & D01 \\
\hline & 3.87 & $\Gamma=2.36_{-0.06}^{+0.05}$ & $\cdots$ & $0.91 / 19$ & $1.01^{a}$ & ROSAT (0.1-2.4) & S98 \\
\hline & 3.7 & $\Gamma=2.33 \pm 0.06$ & $\ldots$ & $1.12 / 79$ & $1.16 \pm 0.05$ & ROSAT (0.1-2.4) & S97 \\
\hline $0836+299$ & 4.1 & $\Gamma=1.7$ & $\ldots$ & $\ldots$ & $0.03^{c}$ & Einstein $(0.2-4.5)$ & BM91 \\
\hline $0838+133$ & 16.5 & $\Gamma=1.22_{-0.05}^{+0.06}$ & $\ldots$ & $1.11 / 182$ & $0.11 \pm 0.01$ & Chandra (0.5-8) & B01 \\
\hline \multirow[t]{2}{*}{$1055+018$} & 3.45 & $\Gamma=2.20 \pm 0.16$ & $\ldots$ & $0.99 / 14$ & $0.21 \pm 0.03$ & ROSAT (0.1-2.4) & S97 \\
\hline & 1.62 & $\Gamma=1.64_{-0.43}^{+0.47}$ & $\ldots$ & $0.85 / 10$ & 0.28 & ROSAT (0.1-2.4) & S98 \\
\hline $1150+497$ & 2.1 & $\Gamma=2.14 \pm 0.05$ & $\ldots$ & $0.93 / 67$ & $0.55 \pm 0.03$ & ROSAT (0.1-2.4) & S97 \\
\hline $1354+195$ & 5.0 & $\Gamma=1.5$ & $\ldots$ & $\ldots$ & $1.19 \pm 0.36^{c}$ & Einstein $(0.2-4.5)$ & B87 \\
\hline \multirow[t]{5}{*}{$1510-089$} & 8.7 & $\Gamma=1.60_{-0.35}^{+0.39}$ & $\ldots$ & $0.44 / 12$ & $0.87 \pm 0.23$ & EXOSAT (2-10) & G95 \\
\hline & 7.6 & $\Gamma=1.89_{-0.17}^{+0.16}$ & $\cdots$ & $1.30 / 26$ & $0.66 \pm 0.05$ & ROSAT (0.1-2.4) & S97 \\
\hline & 6.80 & $\Gamma=1.92 \pm 0.15$ & $\cdots$ & $0.97 / 28$ & 0.72 & ROSAT (0.1-2.4) & S98 \\
\hline & 9.8 & $\Gamma=1.4 \pm 0.1$ & $\begin{array}{l}E_{\ell}=4.95 \pm 0.25 \\
\sigma=0.1\end{array}$ & $0.97 / 50$ & 0.90 & EXOSAT (2-10) & S90 \\
\hline & 7.8 & $\begin{array}{l}\Gamma_{\text {SOFT }}=2.65 \pm 0.63 \\
\Gamma_{\text {HARD }}=2.65 \pm 0.63\end{array}$ & $E_{0}=1.3 \pm 0.3$ & $0.68 / 63$ & $0.53^{b}$ & BeppoSAX (0.4-100) & T00 \\
\hline \multirow[t]{2}{*}{$1641+399$} & 0.9 & $\Gamma=1.98 \pm 0.08$ & $\cdots$ & $1.50 / 44$ & $0.38 \pm 0.03$ & ROSAT (0.1-2.4) & S97 \\
\hline & 1.0 & $\Gamma=1.7$ & $\cdots$ & $\cdots$ & $0.47^{c}$ & Einstein (0.2-4.5) & BM91 \\
\hline \multirow[t]{3}{*}{$1928+738$} & 11.67 & $\Gamma=2.33_{-0.17}^{+0.21}$ & $\cdots$ & $0.83 / 82$ & $1.17 \pm 0.08$ & ROSAT (0.1-2.4) & S97 \\
\hline & 10.4 & $\Gamma=2.1 \pm 0.3$ & $\cdots$ & $1.05 / 30$ & $1.54^{a}$ & EXOSAT (2-10) & L92 \\
\hline & 13.0 & $\Gamma=2.25 \pm 0.48$ & $\cdots$ & $0.97 / 29$ & $0.52 \pm 0.08$ & EXOSAT (2-10) & G95 \\
\hline
\end{tabular}

Columns explanation: 1 = Source IAU name; $2-4=$ Model and parameter values for fits from literature: $N_{\mathrm{H}}=$ absorption column density, $\left(\times 10^{20} \mathrm{~cm}^{-2}\right) ; E_{\ell}=$ Gaussian line energy, in keV; $\sigma=$ Gaussian line width, in keV; $E_{0}=$ break energy between $\Gamma_{\text {SOFT }}$ and $\Gamma_{\text {HARD }}$ in keV; $5=$ Reduced $\chi^{2}$ and degrees of freedom (d.o.f.); $6=$ Flux density at $1 \mathrm{keV}$ in $\mu \mathrm{Jy} ; 7=\mathrm{X}$-ray Instrument used for measurement $/$ sensitivity for the instrument in keV; $8=$ Reference for the reported spectra. B87 = Biermann et al. (1987); BM91 = Bloom \& Marscher (1991); B01 = Brunetti et al. (2001); D01 = Donato et al. (2001); G95 = Ghosh et al. (1995); L92 = Lawson et al. (1992); S97 = Sambruna (1997); S98 = Siebert et al. (1998); S90 = Singh et al. (1990); T00 = Tavecchio et al. (2000).

Notes: $a=$ Monochromatic flux was calculated by us; $b=$ Monochromatic flux density given at $2 \mathrm{keV} ; c=$ Values for $N_{\mathrm{H}}$ and $\Gamma$ are fixed.

This is at odds with the recent claim (Hardcastle et al. 2002 and references therein) that FRIs lack intrinsic X-ray absorption. Suggested scenarios explain the lack of column density by considering that FRIs do not have a molecular torus obscuring the isotropic core emission, or that the inner jet contributes to the $\mathrm{X}$-ray emission on scales larger than the torus. Corroborating evidence for these scenarios was provided by optical studies with HST of a sample of FRIs (Chiaberge et al. 2000), showing a high detection rate of nuclear point sources.

Our findings for 0836+299 (and for other FRIs studied at $\mathrm{X}$-rays by our group) show that at least some FRIs possess excess X-ray absorption. The optical extinction implied by the $\mathrm{X}$-ray column density is $A_{V} \sim 240 \mathrm{mag}$, using Galactic gas-todust ratios $\left(A_{V} \sim 5 \times 10^{-22} N_{\mathrm{H}} \mathrm{mag} / \mathrm{cm}^{-2}\right)$. A nuclear dust lane has been observed in $0836+299$ in ground-based optical images (van Breugel et al. 1986) and in our HST images (Scarpa et al. 2003). In fact, the source has a flat $\alpha_{\mathrm{ox}}$ index and is a luminous IR source detected with IRAS at 25 and $60 \mu \mathrm{m}$ (Golombek et al. 1988), indicating the presence of dust.

The amount of reddening for the optical nuclear light is $A_{V}=1.2 \mathrm{mag}$ (van Breugel et al. 1986). This is much less than the reddening derived from our X-ray column. Therefore, it seems likely that at least part of the X-ray absorber originates in a different component than the gas responsible for the optical reddening (unless that gas-to-dust ratio is much different from Galactic; e.g., Maiolino et al. 2001). The origin of the X-ray absorbing medium in $0836+299$, as in other FRIs studied by us (Sambruna et al. 2003), is not clear. For large inclinations $\left(\gtrsim 80^{\circ}\right)$, a natural possibility is the molecular torus. Indeed, the column density we measure for $0836+299$ is consistent with edge-on Seyfert 2s (Risaliti et al. 1999), and the small value of $R_{i}$ in Table 1 for this galaxy suggests large inclinations. Alternatively, it was proposed that the X-ray absorbing medium is photoionized gas inside the torus (Weingartner \& Murray 2002).

Independent of the origin of the X-ray absorber in $0836+299$, the existence of intrinsic obscuration of FRIs remains a controversial topic, at best. A larger sample of FRIs observed with Chandra and XMM-Newton is needed to address this issue, which would complement ongoing HST studies by other groups (Chiaberge et al. 2002).

Acknowledgements. We are grateful to M. Gliozzi and D. Donato for their assistance with the spatial analysis. JKG and RMS are funded by NASA grants NAS8-39073 and GO 1-2110A, the latter operated by AURA, Inc., under NASA contract NAS 5-26555. CMU acknowledges support from LTSA grant NAG5-9327. CCC acknowledges that radio astronomy at Brandeis University is supported by the NSF. This research has made use of the NASA/IPAC Extragalactic Database 
(NED) which is operated by the Jet Propulsion Laboratory, California Institute of Technology, under contract with the National Aeronautics and Space Administration.

\section{Appendix A: Previous X-ray observations}

In Table 8, we collected the results from previous X-ray observations of the sources of our sample, for comparison with the Chandra spectral results in Tables 4-5.

\section{References}

Antonucci, R. 1993, ARA\&A, 31, 473

Biermann, P. L., Kühr, H., Snyder, W. A., \& Zensus, J. A. 1987, A\&A, 185,9

Bloom, S. D., \& Marscher, A. P. 1991, ApJ, 366, 16

Bridle, A. H., \& Perley, R. A. 1984, ARA\&A, 22, 319

Brunetti, G., Bondi, M., Comastri, A., \& Setti, G. 2002, A\&A, 381, 795

Cheung, C. C., Wardle, J. F. C., Sambruna, R. M., et al. 2003, in preparation

Chiaberge, M., Capetti, A., \& Celotti, A. 2000, A\&A, 355, 873

Chiaberge, M., Macchetto, F. D., Sparks, W. B., et al. 2002, ApJ, 571, 247

Czerny, B., \& Elvis, M. 1987, ApJ, 321, 305

Donato, D., Ghisellini, G., Tagliaferri, G., \& Fossati, G. 2001, A\&A, 375,739

Donato, D., Gliozzi, M., Sambruna, R. M., \& Pesce, J. E. 2003, A\&A, submitted

Elvis, M., Fiore, F., Wilkes, B., McDowell, J., \& Bechtold, J. 1994, ApJ, 422, 20

Eracleous, M., \& Halpern, J. 1998, ApJ, 505, 577

Eracleous, M., Sambruna, R. M., \& Mushotzky, R. F. 2000, ApJ, 537, 654

Fanaroff, B. L., \& Riley, J. M. 1974, MNRAS, 167, 31

Fiore, F., Elvis, M., Giommi, P., \& Padovani, P. 1998, ApJ, 492, 79

George, I. M., Turner, T. J., Yaqoob, T., et al. 2000, ApJ, 531, 52

Ghosh, K. K., \& Soundararajaperumal, S. 1995, A\&AS, 100, 37

Golombek, D., Miley, G. K., \& Neugebauer, G. 1988, AJ, 95, 26

Hardcastle, M. J., Worrall, D. M., Birkinshaw, M., Laing, R. A., \& Bridle, A. H. 2002, MNRAS, 334, 182

Hasenkopf, C., Sambruna, R. M., \& Eracleous, M. 2002, ApJ, 575, 127

Hough, D. H., \& Readhead, A. C. S. 1989, AJ, 98, 1208

Kellermann, K. I., Sramek, R., Schmidt, M., Shaffer, D. B., \& Green, R. 1989, AJ, 98, 1195

Kollgaard, R. I., Wardle, J. F. C., \& Roberts, D. H. 1990, AJ, 100, 1057
Lawson, A. J., Turner, O. R. W., Stewart, G. C., \& Saxton, R. D. 1992, MNRAS, 259, 743

Liu, F. K., \& Xie, G. Z. 1992, A\&AS, 95, 249

Maiolino, R., Marconi, A., Salvati, M., et al. 2001, A\&A, 365, 28

Miley, G. K., \& Miller, J. S. 1979, ApJ, 228, L55

Morganti, R., Killeen, N. E. B., \& Tadhunter, C. N. 1993, MNRAS, 263,1023

Morrison, R., \& McCammon, D. 1983, ApJ, 270, 119

Nandra, K., George, I. M., Turner, T. J., \& Fukazawa, Y. 1996, ApJ, 464,165

Nandra, K., George, I. M., Mushotzky, R. F., Turner, T. J., \& Yaqoob, T. 1997a, ApJ, 488, L91

Nandra, K., George, I. M., Mushotzky, R. F., Turner, T. J., \& Yaqoob, T. 1997b, ApJ, 477, 602

Orr, M. J. L., \& Browne, I. W. A. 1982, MNRAS, 200, 1067

Reeves, J. N., Turner, M. J. L., Pounds, K. A., et al. 2001, A\&A, 365, L116

Reeves, J. N., \& Turner, M. J. L. 2000, MNRAS, 316, 234

Risaliti, G., Maiolino, R., \& Salvati, M. 1999, ApJ, 522, 157

Sambruna, R. M. 1997, ApJ, 487, 536

Sambruna, R. M., Eracleous, M., \& Mushotzky, R. F. 1999, ApJ, 526, 60

Sambruna, R. M., Eracleous, M., \& Mushotzky, R. F. 2002a, NewAR, 46,215

Sambruna, R. M., Maraschi, L., Tavecchio, F., et al. 2002b, ApJ, 571, 206

Sambruna, R. M., Gliozzi, M., Eracleous, M., Brandt, W. N., \& Mushotzky, R. 2003, ApJ, in press [astro-ph/0302506]

Sanders, D. B., Phinney, E. S., Neugebauer, G., Soifer, B. T., \& Matthews, K. 1989, ApJ, 347, 29

Scarpa, R., Urry, C. M., Sambruna, R. M., et al. 2003, in preparation Shastri, P. 1991 MNRAS, 249, 640

Siebert, J., Brinkmann, W., Morganti, R., et al. 1996, MNRAS, 279, 1331

Siebert, J., Brinkmann, W., Drinkwater, M. J., et al. 1998, MNRAS, 301,261

Singh, K. P., Rao, A. R., \& Vahia, M. N. 1990, ApJ, 365, 455

Tavecchio, F., Maraschi, L., Ghisellini, G., et al. 2000, ApJ, 543, 535

Urry, C. M., \& Padovani, P. 1995, PASP, 107, 803

van Breugel, W. J. M., Heckman, T. M., Miley, G. K., \& Filippenko, A. V. 1986, ApJ, 311, 58

Weingartner, J. C., \& Murray, N. 2002, ApJ, in press [astro-ph/0208123]

Wilkes, B. J., \& Elvis, M. 1987, ApJ, 323, 243

Worrall, D. M., Hardcastle, M. J., \& Birkinshaw, M. 2001, MNRAS, 326, L7

Yee, H. K. C., \& Oke, J. B. 1978, ApJ, 226, 753 